\title{
Understanding the impact of advanced PET reconstruction in cardiac PET: The devil is in the details
}

\author{
Ian S. Armstrong, $\mathrm{PhD} \oplus^{\mathrm{a}}$ \\ a Nuclear Medicine, Manchester University NHS Foundation Trust, Manchester, UK
}

Received May 31, 2018; accepted Jun 1, 2018

doi: 10.1007/s12350-018-1329-1

\section{See related article, pp. 1521-1545}

PET-CT continues to see technological advances in both hardware and software which provide improvements to image quality and hence diagnostic confidence. Improvements to detector technology have provided the possibility of Time-of-Flight (TOF) acquisition and reconstruction, which has been commercially available for over a decade. Increases in computational power have allowed the use of more sophisticated iterative reconstruction algorithms that incorporate a more complete modeling of the physics of gamma-ray detection. One particular property that can be modeled is the spatial resolution response of the detector ring to account for degrading depth-of-interaction effects that worsen in magnitude with the increasing radial distance in the field of view. This is commonly referred to as point spread function (PSF) modeling. Image signal-tonoise ratio (SNR) is a fundamental metric of image quality that should be maximized for a given administered activity and image time. In cardiac imaging, it is difficult to quantify the impact of changes in SNR. It is reasonable to assume that an increase in SNR would give the appearance of more uniform perfusion in genuinely normal cases, as the contribution of Poisson image noise to inhomogeneity will be reduced. Both TOF and PSF modeling technologies increase image SNR but understanding the differences of how these two technologies improve image quality when using iterative

Reprint requests: Ian S. Armstrong, PhD, Nuclear Medicine, Manchester University NHS Foundation Trust, Oxford Road, Manchester, UK; ian.armstrong@cmft.nhs.uk, Ian.Armstrong@mft.nhs.uk

J Nucl Cardiol 2018;25:1546-9.

1071-3581/\$34.00

Copyright (C) 2018 American Society of Nuclear Cardiology. reconstruction algorithms is key to knowing how to maximize the benefits from them.

Using scintillation crystals with very short decay time, TOF measures the approximate difference in arrival time of the two $511 \mathrm{keV}$ gamma-rays on the detector ring and thus more accurately localizes the positron-electron annihilation. The current limits of detector technology give an annihilation location within a few centimeters. This acts to confine the distribution of Poisson noise from across the entire line of response to within this area of uncertainty. The implementation of TOF does not require modern iterative reconstruction algorithms, and the original work by Budinger in the early 1980s was performed on filtered backprojection. ${ }^{1}$ The theory showed that, in uniform cylindrical objects, the gain in SNR was proportional to the square-root of the object diameter. In oncology studies, SNR gains from TOF have been demonstrated by measurements in the liver where the patient shape and density is relatively uniform. ${ }^{2,3}$ However, translating SNR gains to the myocardium is not straightforward due to the highly nonuniform shape and density of the patient and also the challenge of defining an appropriate region in which to measure voxel noise. ${ }^{4,5}$

The ubiquitous use of iterative reconstruction adds further complication when assessing SNR gains from TOF as SNR is dependent on the number of updates to the estimated image-usually defined as the product of the number of iterations and subsets. TOF has been shown to accelerate convergence, particularly when measuring contrast recovery of hot spheres in a warm background activity. ${ }^{6}$ Hence, comparing TOF and nonTOF images produced by the same number of image updates may not be appropriate in all situations.

PSF modeling accounts for the gamma-ray interaction in the crystal elements within the block detector arrangement for a PET system. The response of the system to a point source is modeled in the projection operations of the iterative reconstruction process. ${ }^{7}$ The 


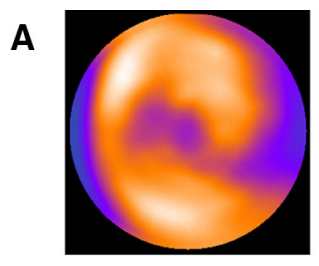

B

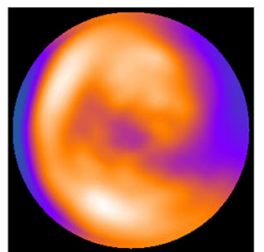

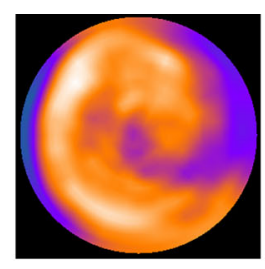
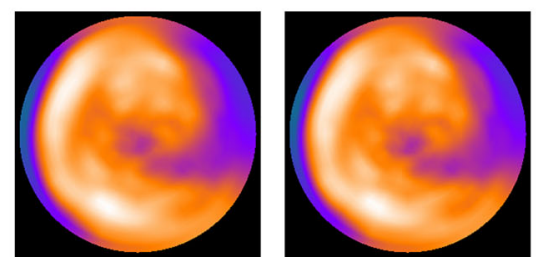
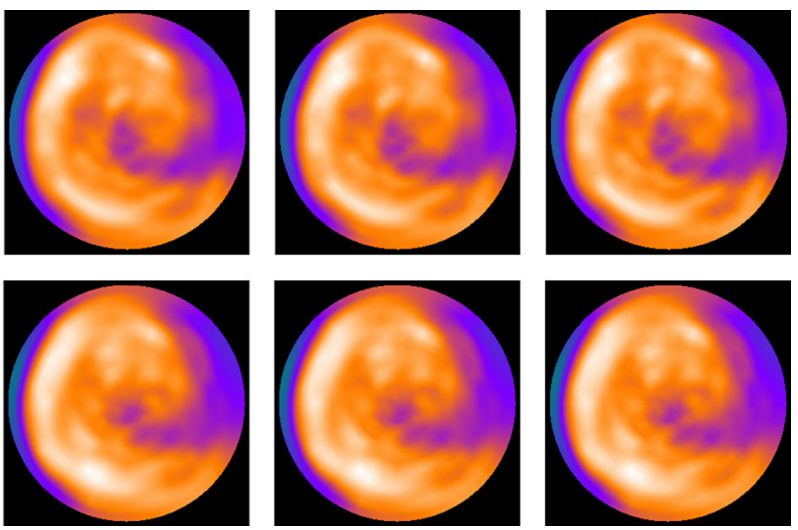

Figure 1. Polar maps of rubidium stress perfusion images for a male patient with a weight of $121 \mathrm{~kg}$ and a BMI of $43 \mathrm{~kg} / \mathrm{m}^{2}$. Images are shown with the increasing numbers of iterations (1 to 6) left-to-right for the following reconstruction algorithms: PSF (A) and PSFTOF (B). PSF algorithms contain 24 subsets, whereas PSFTOF algorithms contain 21 subsets as specified on the Siemens Biograph mCT.

result is improved spatial resolution of images but also a change in the noise characteristics that produces a difference in the noise texture. ${ }^{8}$ This can easily be observed in areas of uniform uptake, such as the liver in FDG oncology images, but the impact of these different noise characteristics for cardiac imaging is not clear. Like TOF, PSF modeling has an impact on the convergence rate but, in contrast to TOF, convergence rates tend to be slower than non-PSF reconstructions. A clear example of this was demonstrated by Le Meunier et al. using a cardiac phantom. ${ }^{9}$ They showed that contrast-to-noise ratio (CNR) of the phantom cardiac insert relative to the bloodpool background was superior using PSF reconstruction compared with the non-PSF reconstruction but required more updates to reach the optimal CNR.

Taking these points of noise reduction and changes to convergence rates into consideration and determining their dependency on patient size is by no means a straightforward task. To address this issue, Dasari et al. ${ }^{10}$ systematically evaluate the impacts of TOF and PSF modeling, both applied individually and also in combination, on rubidium cardiac PET imaging. They look solely at the impact on the static perfusion data and look at differences in segmental uptake and summed stress and rest scores. While their study has a modest number of patients, they apply a very well-thought through method that attempts to separate out the various patient factors that could influence the impact of the various reconstruction options.

As demonstrated in the early TOF theory, the impact of TOF reconstruction is dependent on the crosssectional size of the object or patient in the field of view. In oncology studies, gains in SNR have been shown to be strongly related to patient body mass index (BMI) when measured in the abdomen, but less so when measured in the chest. ${ }^{11}$ Similar findings were also demonstrated when looking at SNR gains from TOF in the myocardium. ${ }^{4}$ This may be because patients carry weight in different places. Male patients with high BMI are likely to have greater amounts of abdominal tissue, whereas female patients are more likely to have greater amounts of breast tissue. Hence, there is likely to be considerable differences in the amounts of tissue in the PET field of view over the heart for male and female patients of similar BMI. This is supported by the authors' observations of the difference in the impact of TOF in male and female patients. No significant differences in perfusion appearance were seen for TOF compared with non-TOF reconstruction in the male patient group. The authors look specifically at the crosssectional area separately to BMI by segmenting the CT image. They show that the impact of TOF increases with the increasing cross-sectional area which is not replicated with the increasing BMI.

The authors are applauded for their complete and effective presentation of images and results-particularly Figure 2-which shows perfusion polar plots for rest and stress images for all four algorithms, allowing the reader to quickly evaluate the differences across the algorithms. A common trend is the improvement in homogeneity of the polar plots when TOF is applied, which is likely due to increased SNR. A more stark observation is the reduction in apparent localized reductions of perfusion when TOF is applied, irrespective of whether PSF is used or not. The most obvious example is patient \#5 that exhibits an antero-septal defect at rest and stress in OSEM and PSF, which normalizes in both TOF and PSFTOF, respectively, giving rise to the possibility that the non-TOF images provide false-positive information. This example is a female 
patient, with both arms in the field of view and hence occupies a considerable portion of the field of view. Other more subtle examples of this phenomenon are patients \#17, \#25, and \#27. The reasoning behind this change in appearance is not clear. As discussed earlier, the impacts of TOF and PSF modeling have been shown to change the convergence rates, and hence, this may be partially attributable to the effect. This is demonstrated in the example shown in Figure 1, acquired at our institution. The figure shows perfusion polar plots for an obese male patient with a BMI of $43 \mathrm{~kg} / \mathrm{m}^{2}$ reconstructed with PSF and PSFTOF algorithms on a Biograph $\mathrm{mCT}$, as used by Dasari et al., with increasing numbers of iterations. In both PSF and PSFTOF images, apical thinning is demonstrated but is more pronounced in the PSF images. However, there is also a subtle apical-septal defect in the PSF images with low numbers of iterations. This defect reduces as the number of iterations increases but is always less apparent when TOF is incorporated.

In their study, the authors use the same number of iterations for TOF and non-TOF reconstructions. However, evidence from phantom and oncology studies has shown that more iterations are usually required for nonTOF reconstructions to provide similar quantitative outcomes. ${ }^{11,12}$ One could hypothesize that if more iterations were performed in either the OSEM or PSF reconstructions, then the defect may begin to normalize and appear more like the images that use TOF. This is supported by data from DiFillipo and Brunken ${ }^{13}$ who show an example of a female patient with a very high BMI of $76 \mathrm{~kg} / \mathrm{m}^{2}$ with an apparent apical-septal defect in non-TOF images that partially normalizes as iterations are increased. The corresponding TOF images show a far-reduced defect.

Dasari et al. demonstrate that the performance of the reconstruction is clearly dependent on multiple factors such as gender, patient size, and setup such as arms up or down. Hence, a thorough evaluation of the impact of the numbers of iterations for the various reconstruction options is not a trivial task, something that they rightfully point out. The lack of ground truth in clinical data to provide validation for evaluating these algorithms remains a problem. Le Meunier et al. only incorporated rest patients when evaluating the impact of PSF modeling; the studies by DiFilippo and Brunken and Dasari et al. are retrospective in approach with no angiographic correlation, hence making it difficult to determine whether apparent perfusion defects in nonTOF images are genuine false-positive cases. Phantom data may help provide a ground truth to some degree, but the structures of most cardiac phantoms do not allow for modeling the varied complexities of both internal and external anatomies in patients. An additional weakness in the current literature is that most, if not all, studies originate from Siemens Biograph mCT systems, and hence, it is not currently possible to translate the findings to systems from other vendors.

\section{CONCLUSIONS}

The work by Dasari et al. has effectively highlighted the impact of advanced PET reconstruction options on rubidium perfusion images. The authors present findings that users would expect to see using typical reconstruction settings used in a clinical scenario in a clear and logical way. It is apparent that TOF has a more substantial impact on image appearance with differences in apparent perfusion defects differing in a small number of their cases. The results presented certainly give scope for follow-up work to more fully understand the interplay of the various technical factors that can influence the images. The addition of ground truth, either phantom studies or angiographic correlation in clinical data, would be highly valued.

\section{Disclosure}

There are no conflicts of interest for this publication.

\section{References}

1. Budinger TF. Time-of-flight positron emission tomography: status relative to conventional PET. J Nucl Med 1983;24:73-8.

2. Akamatsu G, Ishikawa K, Mitsumoto K, Taniguchi T, Ohya N, Baba S, et al. Improvement in PET/CT image quality with a combination of point-spread function and time-of-flight in relation to reconstruction parameters. J Nucl Med 2012;53:1716-22.

3. McDermott G, Chowdhury F, Scarsbrook A. Evaluation of noise equivalent count parameters as indicators of adult whole-body FDG-PET image quality. Ann Nucl Med 2013;27:855-61.

4. Armstrong IS, Tonge CM, Arumugam P. Assessing Time-ofFlight signal-to-noise gains within the myocardium and subsequent reductions in administered activity in cardiac PET studies. J Nucl Cardiol 2017. https://doi.org/10.1007/s12350-017-0916-x.

5. Wells RG, deKemp RA. Does time-of-flight improve image quality in the heart? J Nucl Cardiol 2017. https://doi.org/10.1007/ s12350-017-0992-y.

6. Mettivier G, Salvati R, Conti M, Russo P. The effect of count statistics on the convergence value in OSEM reconstruction in PET and TOF PET. IEEE Nucl Sci Symp Conf Symp 2011; 24006.

7. Panin VY, Kehren F, Michel C, Casey M. Fully 3-D PET reconstruction with system matrix derived from point source measurements. IEEE Trans Med Imaging 2006;25:907-21.

8. Rahmim A, Tang J. Noise propagation in resolution modeled PET imaging and its impact on detectability. Phys Med Biol 2013;58:6945-68

9. LeMeunier L, Slomka PJ, Dey D, Ramesh A, Thomson LEJ, Fracp, et al. Enhanced definition PET for cardiac imaging. J Nucl Cardiol 2010;17:414-26. 
10. Dasari PKR, Jones JP, Casey ME, Liang Y, Dilsizian V, Smith MF. The effect of Time-of-flight and point spread function modeling on ${ }^{82} \mathrm{Rb}$ myocardial perfusion imaging of obese patients. J Nucl Cardiol 2018.

11. Lois C, Jakoby BW, Long MJ, Hubner KF, Barker DW, Casey $\mathrm{ME}$, et al. An assessment of the impact of incorporating time-offlight information into clinical PET/CT imaging. J Nucl Med 2010;51:237-45
12. Jakoby BW, Bercier Y, Conti M, Bendriem B, Townsend D. Physical and clinical performance of the mCT time-of-flight PET/ CT scanner. Phys Med Biol 2011;56:2375-89.

13. DiFilippo FP, Brunken RC. Impact of time-of-flight reconstruction on cardiac PET images of obese patients. Clin Nucl Med 2017;42:e103-8. 